\title{
Hadronic final states in DIS with SHERPA
}

\section{Tancredi Carli}

CERN, Department of Physics, CH-1211 Geneva 23, Switzerland

E-mail: tancredi.carli@cern.ch

\section{Thomas Gehrmann}

Universität Zürich, CH-8057 Zürich, Switzerland

E-mail: thomas.gehrmann@physik.uzh.ch

\section{Stefan Höche*}

Universität Zürich, CH-8057 Zürich, Switzerland

E-mail: shoeche@physik.uzh.ch

\begin{abstract}
We present an extension of the multi-purpose Monte-Carlo event generator SHERPA for processes in deeply inelastic lepton-nucleon scattering. Hadronic final states in this kinematical setting are characterised by the presence of multiple kinematical scales, which were up to now accounted for only by specific resummations in individual kinematical regions. An extension of a known method for merging truncated parton showers with higher-order tree-level matrix elements allows to obtain predictions which are reliable in all kinematical limits.
\end{abstract}

XVIII International Workshop on Deep-Inelastic Scattering and Related Subjects April 19 -23, 2010

Convitto della Calza, Firenze, Italy

\footnotetext{
*Speaker.
} 


\section{Introduction}

Deep-inelastic lepton-nucleon scattering (DIS) offers the possibility to study the structure of the nucleon and the dynamics of strong interactions by means of a pointlike probe. It provides a clean experimental setting to analyse inclusive quantities like for example the proton structure functions. However, the kinematical configurations in DIS are usually very different from those in other processes in collider experiments. The virtuality of the exchanged photon tends to be close to zero, while final state jets might still have large transverse momenta. Experiments conducted at the HERA collider have shown that the large available phase space can lead to a considerable number of hard jets, even if the photon virtuality is low.

This fact poses a certain problem for the simulation of DIS with Monte-Carlo event generators. Such programs usually employ parton showers based on the DGLAP equations [1]. It is assumed that any scattering process factorises into a core $2 \rightarrow 2$ interaction and a shower evolution, which simply "dresses" the hard interaction with softer radiation. As the only hard scale set by the leadingorder DIS process $e^{ \pm} q \rightarrow e^{ \pm} q$ is the photon virtuality, $-Q^{2}$, the probability to produce a jet of transverse momentum larger than $Q^{2}$ would then vanish. In order to reliably simulate DIS events, one must therefore resort to different techniques. We aim at an approach based on combining higher-order tree-level matrix elements with the parton shower along the lines of Ref. [2], which is consistent with the DGLAP framework. The technical prerequisites for realising this method are found in the multi-purpose Monte-Carlo event generator SHERPA [3].

\section{Event generation technique}

The basic idea of the approach is to separate the phase space into a matrix-element and a parton-shower domain through a cut in the phase space of multi-parton processes. The matrixelement domain is then supposed to contain hard, well-separated partons only, while the partonshower domain covers the region where resummation effects become important. Throughout the hard domain parton-shower emissions are corrected using tree-level matrix elements up to a given maximum multiplicity. In the soft domain, the parton shower is applied as is. The separation is achieved in terms of a so-called jet criterion, defining the "hardness" and/or the separation of a parton with respect to others [2]. Several successful studies of various classes of processes have demonstrated the capability of this technique to correctly describe multi-jet final states $[4,5]$.

As pointed out in [2], the above merging algorithm needs to be refined if the scale difference between $Q^{2}$ and the hardness scale $k_{T}^{2}$ of additional partons is large and negative. In this case, logarithmic corrections are not induced by $Q^{2} / q^{2}$, but rather by $k_{T}^{2} / q^{2}$, where $q^{2}$ is the jet resolution scale. The production of the virtual photon can then be regarded as an electroweak splitting process, attached to a core interaction of type $\gamma^{*} j \rightarrow j j$, as depicted in Fig. 1b. In the extreme case of very hard jets, the core process does not even include the virtual photon, cf. Fig. 1c. The main task of the algorithm is to correctly identify the "core" interaction underlying a multi-parton process and to employ it to define starting conditions for the parton shower evolution [6].

Conversely, this idea can be used to lift the restriction on the real-emission phase space at low $Q^{2}$. A similar method is in fact employed in Drell-Yan lepton-pair production via $\gamma^{*} / Z$-exchange, where the separation cut $Q_{\text {cut }}$ between matrix-element and parton-shower domain is set such that 


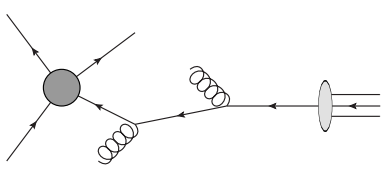

(a)

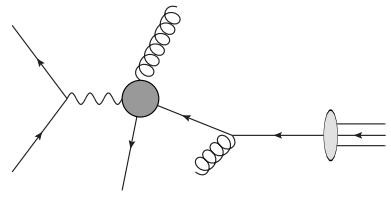

(b)

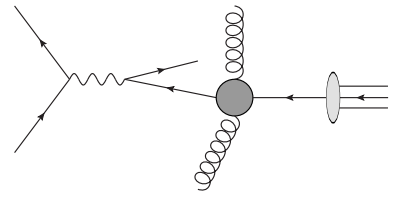

(c)

Figure 1: Schematic view of three possible core process choices in DIS three-jet production. Part (a) corresponds to the most probable core process being the virtual photon exchange, while additional hard partons are interpreted as parton shower emissions. Parts (b) and (c) depict configurations, where the most probable core process is the interaction of the virtual photon with a parton and a pure QCD $2 \rightarrow 2$ process, respectively.

$Q_{\text {cut }}<m_{l l^{\prime}}$, with $m_{l l^{\prime}}$ being the invariant mass of the lepton pair. This choice implies that jets of $k_{T}^{2} \gtrsim m_{l l^{\prime}}$ always fall into the matrix element domain. In deep-inelastic-scattering the situation is slightly different due to the variable value of $Q^{2}$, which plays the role of $m_{l l^{\prime}}$ in the Drell-Yan pair production case. The solution can, however, be identical. We choose

$$
Q_{\mathrm{cut}}=\bar{Q}_{\mathrm{cut}}\left[1+\frac{\bar{Q}_{\mathrm{cut}}^{2} / S_{\mathrm{DIS}}^{2}}{Q^{2}}\right]^{-1 / 2}
$$

where $\bar{Q}_{\text {cut }}$ is a fixed value, much like $Q_{\text {cut }}$ in the Drell-Yan pair production case and $S_{\text {DIS }}<1$ is a constant with lower limit enforced by experimental requirements. $\bar{Q}_{\text {cut }}$ ensures that high- $Q^{2}$, medium- $E_{T, B}^{2}$ events are described by matrix elements, rather than by the parton shower. At the same time, the factor in the square bracket enforces low- $Q^{2}$, high- $E_{T, B}^{2}$ events to be in the matrixelement domain as well, such that the complete available real-emission phase space can be filled.

\section{Comparison with experimental data}

In this section, we show some comparison with experimental data to exemplify the performance of the Monte Carlo simulation. The correct description of the selected measurements is quite challenging for the Monte Carlo traditionally used in the analysis of HERA data [7].

A crucial observable is given by the inclusive jet cross section, differential with respect to $E_{T, B}^{2} / Q^{2}$, where $E_{T, B}$ is the jet transverse energy in the Breit frame. For $E_{T, B}^{2} / Q^{2}>1$ it probes a part of the phase space where leading order Monte-Carlo models without the inclusion of low- $x$ effects are bound to fail in their description of jet spectra. Figure 2a shows that the Monte-Carlo prediction gradually improves with a growing number of final-state partons in the hard matrix elements. The uncertainties associated with a variation of the intrinsic parameters of the merging algorithm are shown in Fig. $2 b$.

It is interesting to investigate jet properties in some more detail. Figure 3 displays rapidity spectra of the forward jet in di-jet production for various regions of $Q^{2}$. As for the case of inclusive jet production we observe a good description of the corresponding $\mathrm{H} 1$ data. 


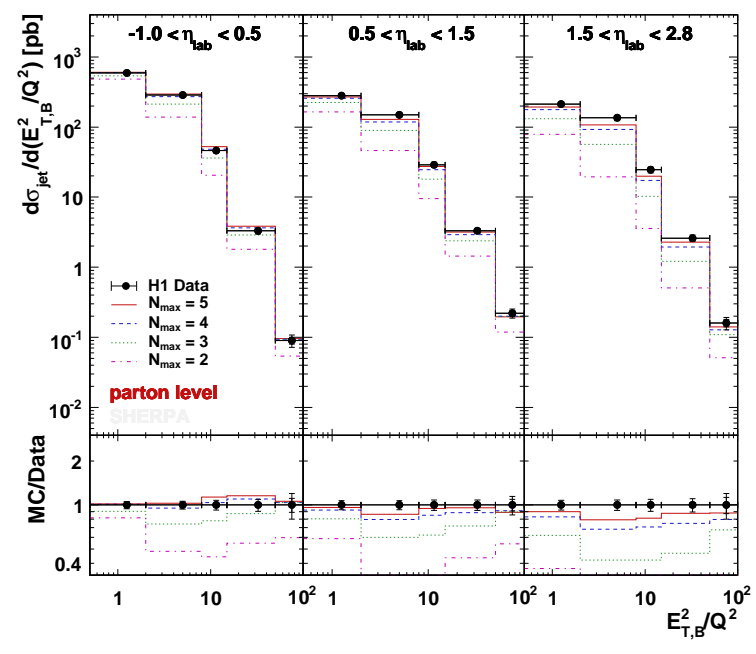

(a)

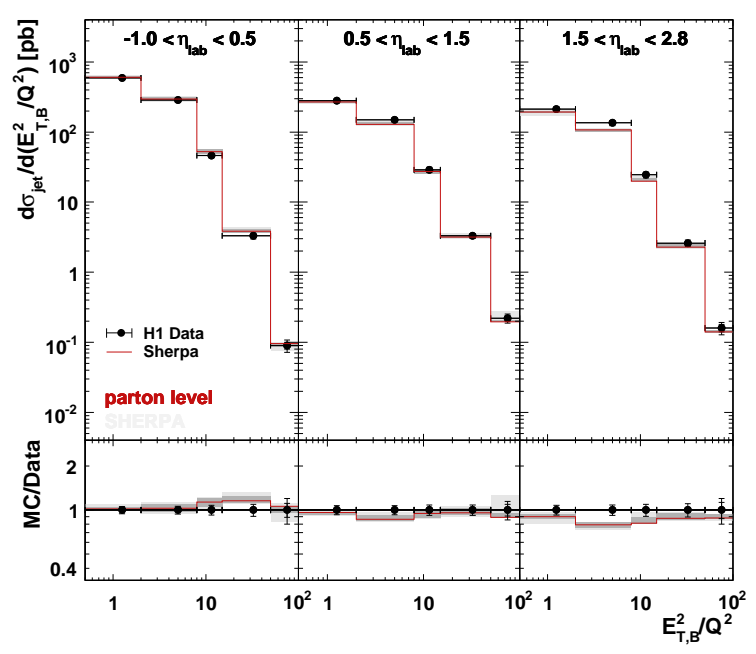

(b)

Figure 2: The inclusive jet cross section as a function of $E_{T, B}^{2} / Q^{2}$ in bins of $\eta_{l a b}$, measured by the H1 Collaboration [8]. $E_{T, B}^{2}$ is the jet transverse energy in the Breit frame, while $\eta_{l a b}$ denotes the jet rapidity in the laboratory frame. Part (a) displays the influence of the maximum parton multiplicity, $N_{\max }$, from hard matrix elements. We show the uncertainty originating from varying $S_{\text {DIS }}$ between 0.5 and 0.7 (light grey band) and from varying $\bar{Q}_{\text {cut }}$ between $3 \mathrm{GeV}$ and $9 \mathrm{GeV}$ (dark grey band) in part (b).

\section{Conclusions}

The SHERPA event-generation framework has been extended to describe hadronic final states in deep-inelastic lepton-nucleon scattering processes. The simulation is based on merging higherorder tree-level matrix elements with a parton shower. When applying this technique to DIS processes, it is vital to correctly identify the core interaction, which can be either electron-quark scattering, photon-parton scattering or a partonic $2 \rightarrow 2$ interaction, depending on the final state kinematics. The particular kinematical situation in DIS also requires to choose appropriate merging scales, depending on the photon virtuality $Q^{2}$. By doing so, we obtain a reliable description of DIS in all kinematical regions, including for example high- $E_{T, B}^{2}$, low- $Q^{2}$ processes.

\section{References}

[1] V. N. Gribov and L. N. Lipatov, Sov. J. Nucl. Phys. 15 (1972), 438-450. L. N. Lipatov, Sov. J. Nucl. Phys. 20 (1975), 94-102. Y. L. Dokshitzer, Sov. Phys. JETP 46 (1977), 641-653. G. Altarelli and G. Parisi, Nucl. Phys. B126 (1977), 298-318.

[2] S. Höche et al., JHEP 05 (2009), 053, [arXiv: 0903 . 1219].

[3] T. Gleisberg et al., JHEP 02 (2004), 056, [hep-ph / 0311263 ]. T. Gleisberg et al., JHEP 02 (2009), 007, [arXiv:0811.4622]. 

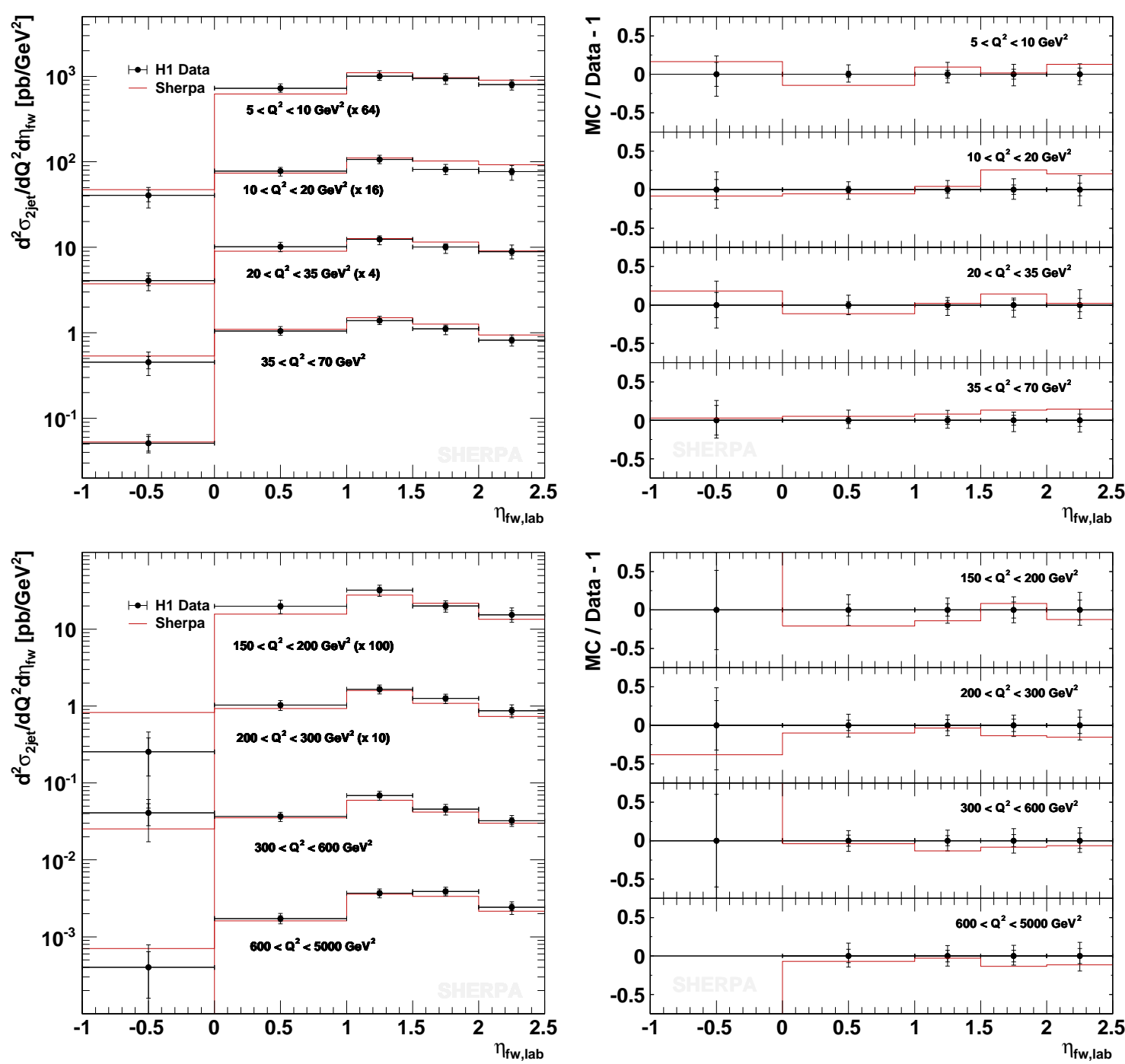

Figure 3: The di-jet cross section as a function of $\eta_{\mathrm{fw}, \mathrm{lab}}$ in bins of $Q^{2}$, measured by the H1 Collaboration [9].

[4] J. Alwall et al., Eur. Phys. J. C53 (2008), 473-500, [arXiv : 0706 .2569]. M. L. Mangano et al., JHEP 01 (2007), 013, [hep-ph/ 061112 9]. J. Alwall et al., JHEP 02 (2009), 017, [arXiv: 0810.5350].

[5] S. Höche, S. Schumann and F. Siegert, Phys. Rev. D81 (2010), 034026, [arXiv: 0912 . 3501].

[6] T. Carli, T. Gehrmann and S. Höche, Eur. Phys. J. C67 (2010), 73, [arXiv : 0912 . 3715].

[7] N. Brook et al., In Hamburg 1995/96, Future physics at HERA, vol. 1, 613-619. N. H. Brook et al., hep-ex/ 9912053 , In Hamburg 1998/1999, Monte Carlo generators for HERA physics, 10-35.

[8] C. Adloff et al., H1 collaboration, Phys. Lett. B542 (2002), 193-206, [hep-ex/ 0206029 ].

[9] C. Adloff et al., H1 collaboration, Eur. Phys. J. C19 (2001), 289-311, [hep-ex/ 0010054 ]. 